\title{
EXPERIMENT OF X-RAY SOURCE BY 9.4 GHz X-BAND LINAC FOR NONDESTRUCTIVE TESTING SYSTEM
}

\author{
Takuya Natsui*, Tomohiko Yamamoto, Fumito Sakamoto, Akira Sakumi, Katsuhiro Dobashi, \\ Mitsuru Uesaka, Nuclear Professional School, the University of Tokyo, Ibaraki, Japan \\ Eiji Tanabe, Naoki Nakamura, AET Japan, Inc., Kawasaki-City, Japan \\ Mitsuo Akemoto, Shigeki Fukuda, Toshiyasu Higo, Noboru Kudoh, \\ Toshikazu Takatomi, Mitsuhiro Yoshida, KEK, Ibaraki, Japan
}

\section{Abstract}

We are developing a compact X-ray source for Nondestructive Testing (NDT) system. We aim to develop a portable X-ray NDT system by $950 \mathrm{keV}$ X-band linac to realize on-site inspection. We use a low power $(250 \mathrm{~kW})$ magnetron as RF source for compactness of whole system. By using low power magnetron, we can use small magnetron power supply and cooling system. Additionally, the system has X-band linac and it has small spot size of electron beam. Our final goal of X-ray spot size is less than $1 \mathrm{~mm}$.

We have designed the linac structure of the $\pi$ mode at low energy parts and the $\pi / 2$ mode at high energy parts by using calculation codes. It was finished to measure resonant frequency and electromagnetic field of the linac. And the result of measurement consists with calculation data.

The components of this system was completed and installed in the Nuclear Professional School, the University of Tokyo. We are carrying out electron beam accelerate testing.

\section{INTRODUCTION}

Nondestructive testing (NDT) is used for inner check of industrial products. There are many methods of NDT. For example, ultrasonic, radiation, neutron and eddy-current. We obtain transfer imaging by X-ray irradiation in an X-ray NDT. The X-ray NDT is most visible and comprehensive.

We are developing the compact X-ray NDT system $[1,2]$. Our system accelerates electron beam in an X-band linac and collides electron with metal target. Then it generates X-ray by bremsstrahlung radiation. Our final aim is to fit in whole system to two suitcase size and carry it anywhere. Figure 1 is the rendering of our proposal NDT system.

A conventional X-ray NDT system has S-band linac and needs large RF source. This device is too large to do onsite inspection. Additionally its electron beam spot size is about $3 \mathrm{~mm}$ and lack of spatial resolution.

Therefore we adopt $9.4 \mathrm{GHz} \mathrm{X}$-band linac and $250 \mathrm{~kW}$ magnetron for RF source. Since a wavelength of X-band

\footnotetext{
* The present study has been performed under the program of KEK to support universities in accelerator developments, and Local Area Consortium Research and Development Project of Ministry of Economy, Trade and Industry.
}

is a quarter of S-band, the linac is smaller. And our magnetron size is very small (about $10 \mathrm{~cm} \times 20 \mathrm{~cm} \times 20 \mathrm{~cm}$, $7.5 \mathrm{~kg})$ as well as low power $(250 \mathrm{~kW})$. Power supply and cooling system can be small because of low power RF. Thus we can design the compact X-ray source.

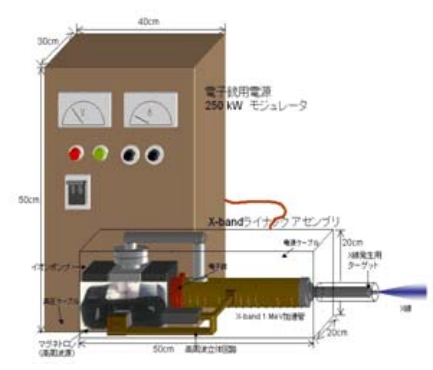

Figure 1: Schematic view of the X-ray NDT system

\section{SYSTEM SUMMARY}

The system consists of the magnetron, microwave components, pulse modulator, thermionic $20 \mathrm{kV}$ electron gun, X-band linac, target for X-ray generation, and control system. The total system size is two boxes of $50 \mathrm{~cm} \times 30 \mathrm{~cm} \times 30 \mathrm{~cm}$ for power supply, $50 \mathrm{~cm} \times 30 \mathrm{~cm} \times 30 \mathrm{~cm}$ for magnetron, linac, cooling system and metal target of X-ray generation.

Figure 2 is a schematic view of whole system. High

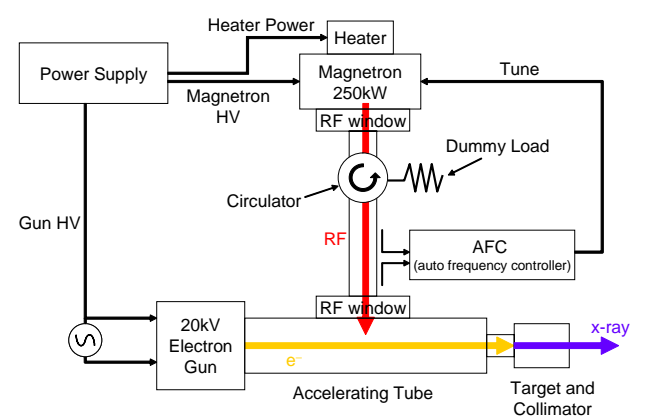

Figure 2: Schematic view of whole system

voltage and heater power is supplied to both the gun and magnetron from the power supply. $20 \mathrm{kV}$ electron beam 
is generated by the gun. This beam current is $400 \mathrm{~mA}$. The magnetron feeds RF to accelerating tube, and electromagnetic field is generated in accelerating tube. And the electron beam is accelerated to $950 \mathrm{keV}$. Finally, the electron beam collide to metal target and generates X-ray by bremsstrahlung.

We adopt an auto frequency controllar (AFC) $[3,4,5]$ because magnetron has instability with a self-oscillation origin. The magnetron frequency is adjusted automatically and stable by the AFC.

\section{DESIGN OF ACCELERATING CAVITY}

In this system, the RF power is only $250 \mathrm{~kW}$. We have to accelerate beam efficiently by low power. The accelerating cavities are designed by using calculation codes SUPERFISH and GPT. We adopt standing-wave APS (Alternative Periodic Structure) cavity of $\pi / 2$ mode for easy manufacturing at base. But according to the simulation, the beam was not accelerated by only $\pi / 2$ mode cavities.

This problem is caused by low speed area. The electron gun generate $20 \mathrm{keV}$ electrons so that the first speed of electron beam is $\beta=0.27$. $(\beta=v / c$ where $v$ is speed of electron. $c$ is speed of light) On the other hand, the most low speed cavity is $\beta=0.4$. The cavity for the more low speed is needed. However, it is difficult to realize a more low speed cavity, which attributes to manufacturing problems. The low speed cavity has narrow width and be not able to manufacture.

Therefore we adopt three $\pi$ mode cavities in the low speed area instead of $\pi / 2$ mode cavity. Finally we designed accelerating tube which has $\pi$ mode and $\pi / 2$ mode cavities. And we simulate beam dynamics in accelerating tube by using GPT. As a result, about $20 \%$ particles are accelerated to more than $800 \mathrm{keV}$. Figure 3 is result of simulation, which is energy spectrum and spatial distribution of electron beam after accelerating.

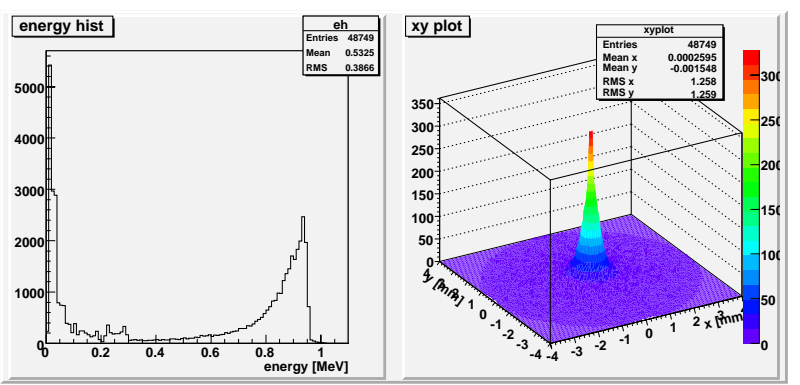

Figure 3: The result of simulation of beam dinamics. A left graph is energy spectrum of beam. A right graph is spatial distribution of beam.

\section{ESTIMATE X-RAY DOSE}

We estimated X-ray dose by using Monte Carlo code of EGS5. In this simulation, we input data which is result of
GPT to EGS5. And X-ray generation by bremsstrahlung is calculated.

The metal target is plates of tungsten and copper which is shown Figure 4. And Figure 5 is a result of EGS5 simulation.

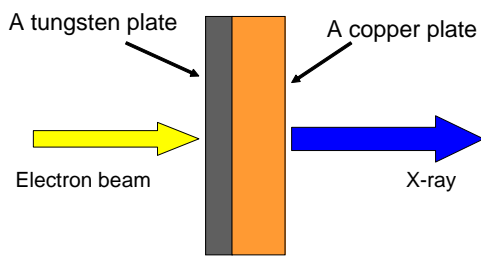

Figure 4: The metal target

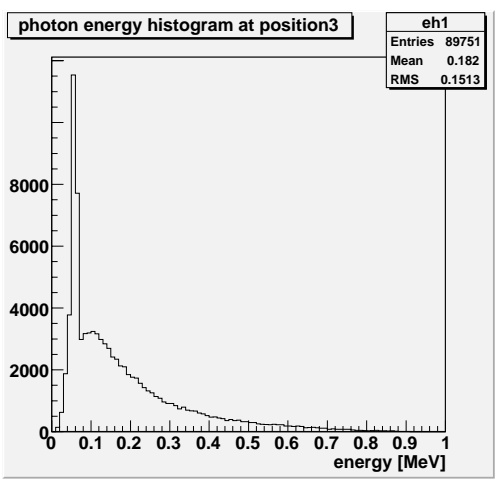

Figure 5: A result of EGS5 simulation. Photons count of generated X-ray.

\section{MANUFACTURING AND MEASURMENT}

The 9.4GHz X-band on-axis coupling standing-wave accelerating tube which has $\pi$ mode and $\pi / 2$ mode APS cavities was made at KEK. And its frequency, Q-value and electric field was measured. Figure 6 is the accelerating tube welded the gun and RF window.

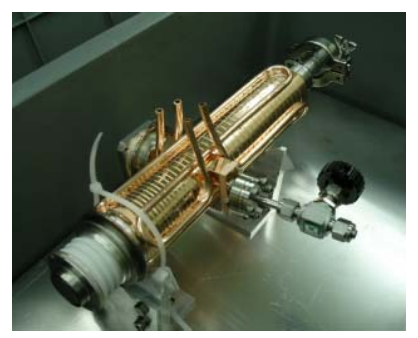

Figure 6: The accelerating tube with gun and RF window

We confirmed that a characteristic of accelerating tube correspond with designed value. Table 1 is the measurement frequency and Q-value. 
Table 1: Cal. \& Meas.

\begin{tabular}{ccc}
\hline & Cal. & Meas. \\
\hline Frequency [GHz] & 9.4001 & 9.3999 \\
Q-value & 6609 & 6618 \\
\hline
\end{tabular}

The electric field of accelerating tube on axis was measured with Bead-Pull measurement. Figure 7 is electric field graph of calculation data and measurement data. The measurement data corresponded with the calculation data.

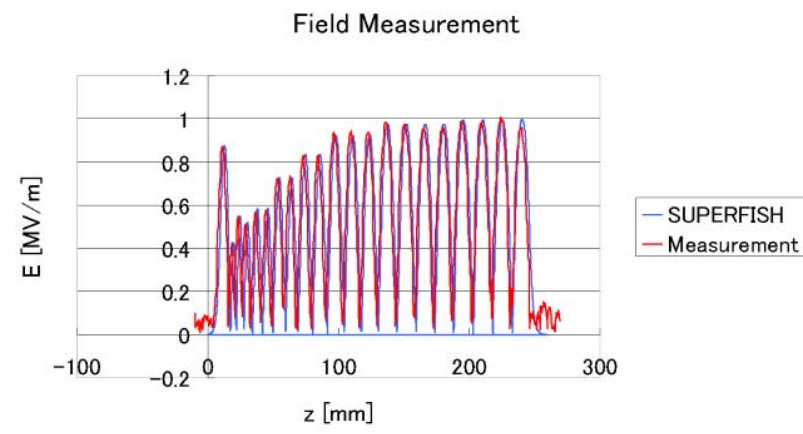

Figure 7: The electric field of accelerating tube on axis. Blue line is calculation data by SUPERFISH. Red line is measurement data. There are correspond.

We had finished a gun emission testing and RF aging at KEK. Figure 8 is wavefome of RF aging.

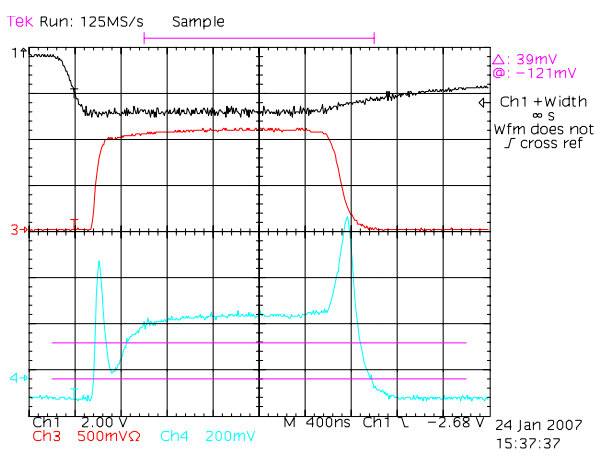

Figure 8: Wavefome of RF aging. Black line is HV of the gun. Red line is incident waveform. Blue line is reflection waveform.

\section{BEAM MEASURMENT}

All component accelerating tube, magnetron, AFC and power supply were installed the University of Tokyo. And we are carrying out electron beam acceleration testing. We are measuring

1.beam current.

2.beam spot size.

3.beam energy spectrum.
A beam current measurement line is constructed and now beam current being measured. Figure 9 is the beam current measurement line at the University of Tokyo. The

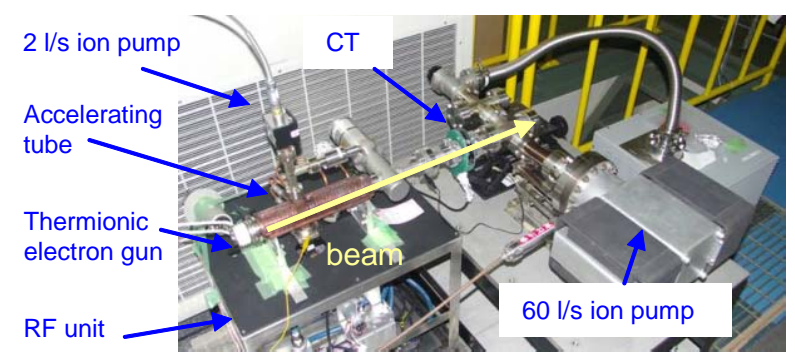

Figure 9: The current mesurement beam line at the University of Tokyo

beam spot size and energy spectrum measurement is scheduled July and August.

\section{CONCLUSION}

We have designed and developed a compact Nondestructive X-ray Testing system using the $9.4 \mathrm{GHz} \mathrm{X}$-band linac with a $250 \mathrm{~kW}$ magnetron. The on-axis coupling standingwave accelerating tube which has $\pi$ mode and $\pi / 2$ mode APS cavities was designed. We confirmed that $20 \%$ electrons are accelerating to more than $800 \mathrm{keV}$. And X-ray generation was estimated by using EGS5.

By using X-band linac and low power magnetron, the accelerator length becomes shorter, and the RF heat loss is remarkably reduced. Therefore the cooling system and power supply becomes smaller, and the total system size becomes more compact and portable. With this NDT system, we can carry out on-site testing of industrial products at various plants and petrochemical complexes.

The total system is constructed at Nuclear Professional School, the University of Tokyo. We are measuring electron beam characteristics. And after that, the X-ray generation testing will be carrying out.

\section{REFERENCES}

[1] T. Yamamoto, et al., "Design of $9.4 \mathrm{GHz} 950 \mathrm{keV}$ X-band Linac for Nondestructive Testing" Proc. of EPAC06 June 26 - 30, 2006, Edinburgh, Scotland, WEPCH182

[2] T. Yamamoto, et al., "Compact $950 \mathrm{keV} \mathrm{X-band} \mathrm{(9.4GHz)}$ Linac X-ray Source for On-site Non-destructive Evaluation" Proc. of IVEC2007 May 15-17, 2007, Kitakyushu, Japan

[3] T. Nishikawa., The Review of Scientific Instruments Volume 37. Number 5 May 1966 pp.652-661

[4] D.E.Nagle et al., The Review of Scientific Instruments Volume 38. Number 11 November 1967 pp.1583-1587

[5] E.A.Knapp et al., The Review of Scientific Instruments Volume 39. Number 7 July 1968 pp.979-991 